\title{
Nouveaux modes de coordination des acteurs dans le développement local: cas des zones rurales au
} Bénin

\author{
Bernard G. Hounmenou
}

\section{(2) OpenEdition \\ 1 Journals}

Édition électronique

URL : http://journals.openedition.org/developpementdurable/1094

DOI : 10.4000/developpementdurable.1094

ISSN : $1772-9971$

Éditeur

Association DD\&T

\section{Référence électronique}

Bernard G. Hounmenou, « Nouveaux modes de coordination des acteurs dans le développement local: cas des zones rurales au Bénin », Développement durable et territoires [En ligne], Dossier 2 | 2003, mis en ligne le 01 octobre 2003, consulté le 01 mai 2019. URL : http://journals.openedition.org/ developpementdurable/1094 ; DOI : 10.4000/developpementdurable.1094

Ce document a été généré automatiquement le 1 mai 2019.

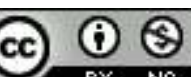

Développement Durable et Territoires est mis à disposition selon les termes de la licence Creative Commons Attribution - Pas d'Utilisation Commerciale 4.0 International. 


\title{
Nouveaux modes de coordination des acteurs dans le développement local: cas des zones rurales au Bénin
}

\author{
Bernard G. Hounmenou
}

1 La décennie 1980 a marqué un nouveau tournant dans l'orientation et la conduite des politiques publiques de développement, notamment au niveau local.

2 Face à l'incapacité grandissante de l'Etat central, à faire face à ses engagements vis-à-vis de ses populations, et aux résultats décevants de ses politiques dirigistes de conduite du développement, les décideurs politiques de nombre de pays en développement se sont vus obligés, sous la contrainte des bailleurs internationaux, de procéder à de profondes réformes sur la gestion de leurs nations. Au nombre des objectifs visés dans le cadre des réformes, on compte entre autres la responsabilisation des populations locales dans la gestion de leurs affaires, notamment par le mécanisme de la décentralisation. L'un des buts visés à travers la mise en œuvre de la décentralisation, est la promotion du développement local sur l'espace des collectivités territoriales. Cet enjeu fait appel à de nouveaux modes de répartition des responsabilités et de conduite des affaires, ce qui fait intervenir la notion de gouvernance au plan local.

3 Le présent article se propose d'aborder cette notion au plan théorique et pratique, en prenant appui sur des expériences en cours dans deux départements de la République du Bénin, un pays qui fait l'objet, depuis peu de temps, de la mise en œuvre du processus de la décentralisation. En effet, confronté à la crise économique aiguë des années 1980, ce pays, dirigé depuis 1974 par un régime politique militaro-marxiste, n'a pas eu d'autres choix que de recourir à la conférence nationale historique des forces vives de février 1990. Les résolutions de cette conférence prônent, entre autres, le libéralisme économique et la promotion de la démocratie participative, à travers le multipartisme intégral et la mise en œuvre du mécanisme de la décentralisation.

4 En prélude à ce processus qui n'a connu sa phase de concrétisation qu'avec l'installation des élus locaux en début 2003, de nombreuses initiatives ont vu le jour dans certaines 
localités du pays, dans le cadre de la promotion de la gouvernance et du développement local. L'objectif visé par endroit, est de préparer les populations locales béninoises, au contexte de la décentralisation.

5 Les éléments empiriques de l'article sont issus de l'étude de cas des communes d'Allada et de Toffo dans le département de l'Atlantique au Sud, et des communes de Glazoué et de Savalou dans le département des Collines au centre du pays.

6 Dans le développement de l'article, une analyse théorique sera faite sur le concept de la gouvernance locale et sur ses liens avec la proximité. La notion de la décentralisation sera abordée et une articulation en sera établie avec la théorie des préférences révélées (théorie des biens publics) d'une part, et avec le type de gouvernance mis en exergue dans les collectivités locales béninoises d'autre part. Enfin, sur la base d'expériences de terrain observées sur les territoires précités, les formes de gouvernance en cause dans les dynamiques participatives locales de développement en zones rurales béninoises seront abordées. Ces expériences feront l'objet d'une analyse sous l'angle de divers aspects tels la révélation des préférences en développement des populations villageoises, les relations de proximité au sein des composantes de celle-ci et l'articulation avec le processus de la décentralisation.

1- Le concept de la gouvernance

7 Depuis quelques années, le thème de la gouvernance comme recherche de nouveaux modes d'organisation territoriale favorisant la participation des habitants et de la société civile, inonde les colloques et les débats, le plus souvent en lien avec les nouvelles recompositions territoriales. Quelques raisons et motivations sont mises en avant pour rechercher la participation de la société civile. Parmi celles-ci figurent notamment :

- - Une conception moderne du management local, de façon à prendre en compte les attentes des populations et accroître leur degré de satisfaction, et pour donner une image ouverte et moderne de la gestion locale.

- - Un souci de renouveau de la démocratie pour montrer une autre image de la politique, lutter contre la désaffection qui la touche et réconcilier les citoyens avec les élus.

- - La nécessité pragmatique de faire adhérer les acteurs concernés par les politiques mises en œuvre, pour définir avec eux les objectifs de développement, pour préparer les décisions et pour agir en partenariat avec eux.

8 Ces dernières années sont en effet marquées par des changements notables dans la conception de l'action publique, qui se sont traduits par un déplacement des analyses de la seule action des gouvernements à des formes de mobilisation d'acteurs privés (associatifs, entreprises, simples citoyens,...), contribuant collectivement à la régulation des espaces et des activités.

9 La réflexion sur le gouvernement des territoires, leur modes de gestion et d'administration, est renouvelée par les concepts de gouvernance et de " gouvernance locale ", posant ainsi la question de l'autonomie relative des processus de développement locaux (Bertrand et al., 2001).

10 Pour Yann Bertacchini (op. cit.), le terme de gouvernance renvoie à l'intervention combinée d'acteurs plus ou moins divers sur un territoire de plus en plus vaste, ainsi qu'à la capacité de ce système d'acteurs de produire des politiques publiques cohérentes sur l'espace. Elle fait appel à la fois, à une coordination des actions entreprises par les acteurs, ce qui nécessite donc un processus de synchronisation tel que la planification, 
mais aussi à une cohérence qui sous-entend la reconnaissance des finalités communes à atteindre.

11 La gouvernance se définit selon Bird et Vaillancourt (1997), par l'exercice de l'autorité et du contrôle au sein d'une société dans le cadre de la gestion de ses ressources en vue du développement social et économique.

Elle ne se réduit pas à l'action des institutions gouvernementales nationales; elle implique leur interaction avec non seulement les différents niveaux territoriaux du gouvernement, mais également l'ensemble des organisations représentatives de la société civile ainsi que le secteur privé, qui doivent s'engager dans un partenariat pour le développement (Shah, 1998).

13 Selon Le Galès (1995), la gouvernance se définit d'une part comme la capacité à intégrer et à donner forme aux intérêts locaux, aux organisations et groupes sociaux, et d'autre part, la capacité à les représenter à l'extérieur, à développer des stratégies plus ou moins unifiées en relation avec le marché, l'état, les autres villes et autres niveaux gouvernementaux. Il assimile aussi la notion de gouvernance aux interactions entre l'Etat et la société et aux modes de coordination pour rendre possible l'action publique.

dernière assertion du thème renvoie à ce qui se passe au delà d'une organisation, à savoir la capacité à organiser l'action collective, à construire des coalitions et des partenariats orientés vers des buts spécifiques (Body-Gendrot et Le Galès, 1997).

Elle renvoie non seulement à des actions collectives, mais aussi à une responsabilisation des différents acteurs et aux relations de partenariat entre ceux-ci dans le cadre de la promotion économique et sociale $\mathrm{du}$ territoire. Ces actions impliquent un ensemble d'institutions dont les collectivités locales, les services déconcentrés de l'état, les institutions de la société civile ou des milieux professionnels, le secteur privé, ...

La base d'analyse du concept de gouvernance peut être rapportée à deux approches théoriques, dont les origines remontent respectivement en sciences économiques et en sciences politiques. La première s'intéresse particulièrement aux coordinations entre acteurs, alors que la seconde se rapporte au gouvernement des territoires. En sciences politiques, la conceptualisation de la gouvernance territoriale est issue de l'analyse du gouvernement local, et plus spécialement du gouvernement urbain. Le recours à la notion de gouvernance entend avant tout caractériser les activités du gouvernement (ou de régulation) de systèmes complexes ou fragmentés. Dans cet usage, le terme de gouvernance ne se différencie pas toujours de la notion de gouvernement, car l'accent reste porté sur les arrangements institutionnels (et en l'occurrence inter-institutionnels), à l'échelle des grandes agglomérations (Parks et Oakerson, 1989). Cette équivalence est très fréquente dans les travaux américains, pour des raisons de tradition intellectuelle (Bertrand, op. cit.).

17 Au-delà des situations de fragmentation institutionnelle, caractéristiques de la plupart des agglomérations, la notion de gouvernance est également mobilisée pour rendre compte de la pluralité des acteurs associés à la définition de l'action publique, en premier lieu, en milieu urbain.

18 Stone (1989) souligne que l'élection ne suffit pas à donner aux décideurs publics la capacité d'agir ; cette capacité doit être construite dans une relation avec l'ensemble (ou du moins en partie) des acteurs susceptibles de contribuer, à la mise en œuvre des actions envisagées. 
19 Dans le prolongement des travaux américains, l'usage de la notion de gouvernance en Europe, entend souligner la participation de divers intérêts privés au systèmes de décision publiques, régissant les agglomérations, et par extension l'action publique territoriale (Padioleau, 1991).

20 En sciences économiques, le concept de gouvernance est mobilisé tardivement, en particulier en lien à deux débats au cœur de l'analyse du fait productif et des concentrations spatiales d'activités. Le premier porte sur la nature des coordinations entre agents individuels ou collectifs (Commons, 1934), dès lors qu'est rejetée une position standard considérant le seul prix du marché dans la régulation des échanges. Le second est celui de la prise en compte de la dimension spatiale, comme facteur intrinsèque du fait productif.

2- Gouvernance locale et dynamiques de proximité :

21 Les analyses des processus de décision locaux renvoient à des formes d'action collective, reposant sur des jeux de négociation, de compromis, d'alliances, qui supposent l'activation de relations et de réseaux divers. Ces mécanismes peuvent être rattachés à diverses formes de proximité : proximité géographique des acteurs urbains (ou locaux, ou départementaux,...), proximité organisationnelle au sein de réseau d'influence ou d'instances formalisées de concertation, etc. C'est au nom de la proximité géographique que la démocratie participative est prônée. Elle renvoie in fine, à des proximités organisationnelles, soit qu'elle suscite la mise en place de tels dispositifs (conseils de quartiers,...)

22 Il importe ici de préciser au passage, ce qu'est la proximité. Le concept de proximité fait référence à une séparation économique ou géographique entre agents (individuels ou collectifs), et donc à leur éloignement plus ou moins fort.

23 Selon A. Torre et J.-P. Gilly (2000), la proximité a deux composantes majeures: la proximité géographique et la proximité organisationnelle. Ils identifient au sein de cette dernière deux variantes: une logique d'appartenance et une logique de similitude. Gabriel Colletis et ali (1999) séparent assez nettement les deux variantes de la proximité organisationnelle et distinguent alors trois types de proximité: la proximité géographique, la proximité organisationnelle et la proximité institutionnelle. La proximité géographique fait référence à la notion d'espace, renvoyant à la localisation des entreprises. Elle traite de la séparation dans l'espace et des liens en termes de distance. A. Torre et J.-P. Gilly (op. cit) estiment qu'elle doit être appréhendée comme un véritable construit social, où le temps joue un rôle primordial. Elle permet des externalités pécuniaires qui peuvent être favorables à la structuration locale. La proximité organisationnelle traduit la séparation économique entre agents, et traite des liens en terme d'organisation de la production. Elle concerne les interactions entre acteurs (individuel et collectifs), et la dimension collective à l'intérieur ou entre les organisations, reposant sur un cadre cognitif commun. Sont proches, en terme organisationnel, les acteurs qui appartiennent au même espace de rapport (firmes, réseau,...).

24 La proximité institutionnelle lie les acteurs qui se ressemblent ou qui possèdent le même espace de référence, et partagent les mêmes savoirs, si bien que la dimension institutionnelle est importante. Cette proximité est liée à une relation de "ressemblance" des représentations et des modes de fonctionnement. Elle repose donc sur l'adhésion des acteurs à un espace commun de représentations, de règles d'action et de modèle de pensée, orientant les comportements collectifs (Torre et Gilly, 2000). 
La rhétorique de la proximité est au cœur des démarches participatives. Elles postulent en effet, que les programmes répondront d'autant mieux aux besoins réels des populations, qu'ils auront été définis au plus près des bénéficiaires, et autant que possible par eux. Une procédure telle que le budget participatif par exemple, entend ainsi réorienter les programmes publics vers les actions qui auront été jugées prioritairement par les habitants des quartiers concernés.

Dans la gouvernance, la notion de proximité n'est toutefois pas explicitement mobilisée.

7 L'analyse des fondements théoriques, économiques et politiques du concept de gouvernance met en évidence une relation proximité - gouvernance, qui ne tient pas tant au rapport de "cause à effet " mais au rapport de convergence d'analyse. Les seuls critères de proximité tant géographiques qu'organisationnels n'engendrent des phénomènes de gouvernance, que dans certaines conditions, celles-ci étant portées par un projet commun, par un renforcement des relations citoyens/élus, souvent accompagnées d'un «porteur de l'action collective à la connaissance du public » (Unal, 2000). La proximité organisationnelle souligne l'intérêt d'une prise en compte des relations, rapprochant les divers acteurs locaux, à travers les institutions formelles, des réseaux, des relations économiques, ou des rapports d'influence et de dépendance. Sous cet angle, le concept de gouvernance met l'accent sur les liens étroits, qui associent les décideurs publics et les représentants des différents autres acteurs.

Dans les approches économiques, la recherche de théorisation des dynamiques territoriales, par la convergence de différents types de proximité, fait émerger le concept de gouvernance dans l'interprétation du fait productif. Il y a donc une dimension explicative de la gouvernance par les proximités.

L'approche des sciences politiques, centrée sur l'analyse des modes de gouvernance territoriaux, ne traite qu'implicitement les dimensions de la proximité, révélant ainsi une convergence d'intérêt pour ces deux objets que sont la gouvernance et les proximités. Ainsi, l'existence de formes de proximité est une condition de la gouvernance, mais ne saurait en être la cause suffisante.

30 Au plan pragmatique, un paradoxe est à l'origine des limites manifestes, rencontrées par les politiques publiques qui se réclament de la gouvernance, et qui tentent d'impulser artificiellement des dynamiques de développement territorial. En effet, sur la base d'expériences locales réussies, elles entendent mettre en œuvre des modalités génériques ou systématiques de renforcement volontariste des proximités entre acteurs. Il s'agit d'un passage de la singularité d'itinéraires de développement propres à un territoire, à des modalités particulières de configuration d'acteurs et d'activités, et à des modalités normalisées et universelles d'organisation des acteurs. Il y a une véritable dénaturation des démarches ascendantes qui voudraient systématiser des expériences locales, universaliser le singulier.

31 A cette contradiction qui voudrait imposer des formes d'organisation, dont la richesse provient largement du caractère spontané, injonction descendante à la créativité ascendante, s'ajoute une autre dimension problématique, autour de la dimension temporelle des phénomènes. Les politiques d'encouragement à l'activation des liens de proximité négligent largement la dimension temporelle de construction de ces proximités. Cette construction est pourtant issue le plus souvent de processus d'apprentissages progressifs, d'interactions répétées qui prennent sens dans le temps long. 
32 Ainsi, il apparaît que les ambitions placées dans les politiques publiques de développement, tablant sur l'activation de la proximité révèlent pour une large part du mirage. Si la proximité est bien une opportunité, voir une condition de développement, elle n'en constitue pas la clé systématique (Bertrand et ali, op. cit). La proximité des acteurs sur un territoire peut être à la base de certaines externalités liées à son développement. Elle peut favoriser par exemple un processus de concertation et de dialogue social, qui amène les acteurs à converger dans leurs ambitions pour leur développement. L'activation de cette proximité peut être aussi favorisée par les réformes institutionnelles. C'est notamment, le cas du processus de la décentralisation.

3- La gouvernance et la décentralisation

33 La décentralisation est considérée comme l'une des formes de retrait de l'Etat d'un certain nombre de fonctions, et se situe aujourd'hui dans la perspective de libéralisation des économies, en particulier celles des pays en développement.

Elle consiste en un transfert de pouvoirs, c'est à dire de compétences et de ressources financières nécessaires à son exercice du niveau central (Etat) au niveau local (collectivités territoriales) et à des organes élus.

La décentralisation s'inscrit dans une réflexion sur la gouvernance. Celle-ci implique l'interaction des institutions gouvernementales non seulement avec les différents niveaux territoriaux, mais également avec l'ensemble des institutions représentatives de la société civile ainsi que le secteur privé, qui doivent s'engager dans un partenariat sur le développement (Shah, 1998). Ceci demande dans certains contextes, un changement notable dans les processus de prise de décision et dans la gestion des affaires au plan local.

3.1- Décentralisation et révélation des préférences en développement

36 Assimilé à une amélioration de bien être individuel et collectif, le développement est considéré comme un bien public, c'est-à-dire, un bien dont la consommation ne fait pas l'objet d'exclusivité et de rivalité dans la population. Tout comme dans la consommation des biens privés, ceux-ci peuvent dans la recherche de la satisfaction de leur utilité, révéler leurs préférences en matière de la consommation de ce bien. La question de la révélation des préférences fait l'objet d'une confrontation entre deux théories, celle de l'échange volontaire et celle de la contrainte.

37 La théorie de l'échange volontaire (Wicksell, 1896 ; Lindahl, 1919) encore appelée théorie du bien public ou théorie de juste prix ou encore théorie des préférences révélées est une théorie libérale qui associe rationalité privée et rationalité publique. Cette théorie stipule que dans une économie où il y a des biens privés et des biens publics, l'Etat n'est qu'une entreprise spécialisée dans la fourniture de ces derniers, en fonction de la demande des particuliers. L'équilibre entre offre et demande détermine un prix ou un montant global d'impôts à prélever. Les biens publics sont ainsi payés à un juste prix. Chaque contribuable contribue volontairement par l'impôt au financement de la production étatique, en proportion des satisfactions qu'il retire des biens publics (Jarret et Mahieu, 1998).

38 Cette théorie des biens publics s'oppose à une autre : la théorie de la contrainte, encore appelée théorie du service public ou théorie de la budgétisation. Cette théorie distingue fondamentalement l'économie privée de l'économie publique. Dans cette théorie, c'est l'Etat qui, compte tenu de ses fonctions tutélaires, détermine quels sont les services publics permettant d'assurer à la fois la satisfaction des intérêts particuliers et la 
maximisation de l'intérêt collectif. Dans le cadre d'une économie d'attribution, les services publics sont fournis gratuitement ou contre paiement d'un tarif subventionné. Leur financement est assuré par les contribuables via la budgétisation, indépendamment de l'utilisation effective des services publics (Jarret et Mahieu, 1998).

Ces deux théories ont fait l'objet de critiques pour leur extrême position, d'un côté en faveur des préférences individuelles et de l'autre en faveur de la référence exclusive à l'Etat dans la détermination du bien-être des citoyens. En réalités, les choses ne se passent pas nécessairement ainsi, notamment en ce qui concerne l'expression des préférences collectives.

En effet, l'expression des décisions collectives intervient au bout des transactions qui donnent lieu à des choix qui apparaissent comme une sorte de convention au groupe social concerné.

Ces transactions sont des situations de négociation où jouent conjointement la volonté et les forces économiques et sociales, qui contraignent et organisent le processus de choix (Commons, 1934). Ce dernier indique que l'ordre social correspond à un compromis entre les intérêts et non à l'agrégation des décisions individuelles menées indépendamment les unes des autres, en fonction de variables données par ailleurs. Il correspond à de négociations sur les valeurs et sur le cadre institutionnel dans lequel elles interviennent. Pour lui, l'ordre social est un ordre construit par l'action collective, et non un ordre spontané... Il insiste par ailleurs sur l'intention qui préside aux actions et sur le caractère collectif et négocié des décisions, qu'il oppose à leur simple juxtaposition.

42 Selon Eric Brousseau (1993), si les agents sont nombreux et prennent des décisions indépendantes, alors rien ne garantit qu'ils convergeront vers un équilibre, même s'ils sont parfaitement rationnels. La cohérence des comportements individuels est assurée par des conventions conduisant les agents à choisir, parmi les alternatives rationnelles, des comportements compatibles, les uns avec les autres. Si la convention est convenablement choisie, elle conduira à une situation optimale (Walliser, 1991).

43 Les préférences révélées en matière de décisions collectives, intègrent des aspirations rationnelles des individus sans en être la somme arithmétique. Cet apport de la théorie institutionnelle aux éléments des deux anciennes (celles de la contrainte et celle des biens publics), semble rentrer en articulation avec les externalités de la décentralisation en matière de décision et de préférences collectives, notamment au niveau local. En favorisant la responsabilisation des collectivités locales dans la prise en charge et la gestion de leurs affaires, la décentralisation permet l'émergence et le développement d'un mécanisme de décision collective au niveau territorial. Le rapprochement des populations des mécanismes de prise de décision sur les affaires les concernant, peut permettre à celles-ci, d'exprimer leurs choix et de les faire prendre en compte dans les politiques publiques. Par ailleurs, la proximité entre les administrateurs et les administrés que permet la décentralisation permet l'émergence de processus de dialogue, de concertation et de négociation qui peut jouer en faveur de la prise de décision et la mise en œuvre des politiques qui reflètent les désirs des citoyens. Ainsi, à travers le rapprochement entre pouvoir et populations locales dans le processus de prise de décision, la décentralisation permet à celles-ci de mieux révéler leurs préférences de développement.

Une décentralisation effective et bien réussie impliquant une gestion participative, peut constituer un facteur déterminant le succès d'un processus de développement local, dans 
la mesure où les acteurs locaux peuvent orienter leurs décisions vers la promotion d'activités qui sont propres à leur territoire. Il faut toutefois noter la décentralisation peut sous certaines conditions, conduire à la marginalisation de localités pauvres aux côtés d'autres relativement plus riches. En effet, le dynamisme particulièrement important de certaines localités peut attirer vers celles-ci l'essentiel de l'activité économique, laissant les localités voisines dans un état de pauvreté. Cette situation donne lieu à un déséquilibre dans le développement des localités d'un même environnement.

Par ailleurs, le fait que les élus locaux émanent des choix démocratiques des populations, ne les dédouane pas toujours de toute tentative égoïste pouvant les amener à vouloir tirer des rentes politiques de leur position. En effet, il peut arriver qu'ils profitent de cette position pour opérer des choix égoïstes qui biaisent les aspirations réelles des populations qu'ils représentent. Ainsi, la décentralisation, malgré ce mérite de permettre une prise en compte plus accrue de la volonté des populations dans les choix publics, ne constitue pas cependant une panacée en la matière de révélation de préférences collectives au plan local.

3.2- Décentralisation et gouvernance dans le contexte béninois

Au Bénin, la gestion et l'administration des collectivités locales relevaient dans chaque sous préfecture (jusqu'en fin 2002), de la responsabilité du sous préfet nommé par l'Etat . Celui-ci était sous la tutelle hiérarchique du préfet de département. Le préfet était aussi nommé par l'Etat. Dans l'administration de leurs circonscriptions, les sous préfets recevaient des instructions de leur ministère de tutelle à qui, ils doivent rendre compte au même titre que le préfet. La population à la base était souvent peu concertée dans les prises de décision, compte tenu de son éloignement des niveaux où celles-ci se prennent.

Pour éviter la déconnexion totale des populations de la gestion des sous préfectures, il avait été créé dans chaque sous préfecture un conseil consultatif comprenant le sous préfet, tous les responsables de services déconcentrés de l'Etat (en poste sur le territoire) et un représentant de chaque arrondissement, différent de son chef (ex-maire). Dans les prises de décisions importantes sur la gestion du territoire, le sous préfet se concertait avec ce conseil consultatif, pour recevoir les observations de ses membres.

Dans le cadre de l'avènement des nouvelles collectivités décentralisées, le législateur béninois

a prévu à la place de la sous préfecture, une collectivité autonome appelée commune, composée d'arrondissements. Les territoires de ces derniers correspondent en zones rurales, aux territoires des ex- petites communes. La loi prévoit à la tête de la commune, un conseil d'arrondissement dont les membres sont élus à la suite d'une campagne électorale, par l'ensemble de la population communale en âge de voter. Ce conseil d'arrondissement est dirigé par un maire et des adjoints, désignés de façon démocratique par les membres. Le maire nomme à la tête de chaque arrondissement, un chef choisi parmi les membres du conseil.

50 L'élection des conseillers communaux sur tout l'étendu du territoire béninois, est intervenue en décembre 2003 et l'installation des élus dans leur fonction s'est déroulée entre février et mars 2004. En son article 84, la loi n97-029 du 15 janvier 1999 sur l'organisation des communes, transfère l'autorité de la planification du développement local sur les futures communes. Cet article 84 précise : La commune élabore et adopte son plan de développement. Elle veille à son exécution en harmonie avec les orientations nationales, en vue d'assurer les meilleures conditions de vie à l'ensemble de la 
population. Dans ce cadre, elle élabore les documents de planification nécessaires (le schéma directeur d'aménagement de la commune, le plan de développement économique et social, ...), etc. Pour l'exercice de cette compétence, la loi prévoit la possibilité pour la commune de recourir à des acteurs privés ou publics, en vue de l'exécution des opérations qui découlent des compétences communales.

51 Ainsi en conformité avec les stratégies sectorielles et les réglementations nationales en vigueur, la commune peut en cas de besoin solliciter le concours des services techniques de l'état. Elle peut aussi créer ses propres services techniques. Dans l'exécution des opérations qui en découlent et sous sa maîtrise d'ouvrage, elle peut déléguer, se faire assister, concéder, affermer, sous traiter ou passer des contrats. Elle a recours à cet effet aux services d'Etat, aux sociétés d'économie mixte ou agence d'exécution, aux Organisations Non Gouvernementales (ONG), aux comités de gestion, aux partenaires en développement, aux sociétés privées,...conformément à la réglementation en vigueur.

52 Ainsi, il apparaît la reconnaissance et la prescription d'un partenariat, entre la collectivité locale et les divers acteurs qui sont entre autres l'Etat, les privés et les organisations de la société civile, dans le cadre de la conduite des opérations de développement sur le territoire.

53 Cette loi béninoise n'est pas restée explicite, en ce qui concerne la participation directe des populations à la base au processus de planification du développement local. Toutefois, elle prévoit en son article 131, que le conseil d'arrondissement fait des propositions relatives au développement et à la bonne administration de l'arrondissement. En ce qui concerne le conseil villageois, le texte ne lui accorde explicitement que le pouvoir d'émettre des avis sur les affaires pour lesquelles, il est requis par le conseil d'arrondissement et de faire des propositions relatives à la bonne administration du village ou du quartier de ville.

54 Au regard de ses orientations de la loi et des expériences en cours dans certaines localités du Bénin, notamment dans les Collines et l'Atlantique, il apparaît que d'importants outils d'animation et de gestion de la participation des populations locales à leur développement existe déjà à la portée des nouveaux élus locaux.

Même si jusque là, les interventions directes des collectivités locales en matière de développement ne sont pas observables, les territoires béninois font depuis quelques années, l'objet d'importantes dynamiques dans le domaine. Celles-ci sont en effet, directement portées par les populations, qui s'organisent dans plusieurs agglomérations villageoises à travers différents systèmes locaux d'action publique.

4- Gouvernance et dynamiques de participation locales au développement

Les dynamiques de développement local sur les territoires ruraux au Bénin, mettent souvent en jeu l'intervention de plusieurs acteurs auprès des populations d'une même localité. Ces derniers contribuent à la poursuite d'intérêt collectif des populations dans un même espace administré, ce qui fait référence à la notion de système local d'action publique.

4.1- Gouvernance et système local d'action publique

Les définitions de la gouvernance, en mettant l'accent sur la gestion par un ensemble d'acteurs, de ressources qui leur sont communes, mettent en relief la notion d'action publique.

Beslay C. et als (1998) définit une action publique comme toute opération d'animation, d'aménagement, de financement ou d'autre nature, se donnant comme justification des 
objectifs d'intérêt collectif (dépassant des intérêts individuels d'une personne ou d'une organisation). Les acteurs publics sont des organisations ou des individus se plaçant sur ce registre d'action. Une collectivité territoriale est un acteur public, mais c'est aussi le cas d'une entreprise privée engageant des actions de mécénat ou d'aide sociale.. L'action publique est produite par des acteurs individuels et des organismes dépendant de l'Etat, des collectivités locales ou encore des milieux professionnels.

Dans chaque espace administré, apparaît un système local d'action publique, rassemblant des organisations publiques, para-publiques ou privées, plaçant leurs interventions sur le registre du bien commun et exerçant leur action sur une aire déterminée. La coordination et les formes de régulation des différentes interventions à l'intérieur du système, font appel à la gouvernance locale. Le concept de gouvernance demande donc de s'intéresser aux réseaux locaux, aux groupes sociaux qui sont susceptibles de constituer une localité. L'autorité locale devient un acteur important certes, mais un acteur parmi tant d'autres, tout comme l'Etat (Le Galès, op. cit).

60 En France, la création des conseils de développement a constitué une innovation législative importante, pour donner aux acteurs de la société civile et aux habitants une place et un rôle dans l'élaboration, la conduite et l'évaluation d'une politique de développement territorial. La mise en place d'un conseil de développement, en lien avec l'élaboration, la mise en œuvre et l'évaluation d'un projet de territoire a un impact favorable sur l'émergence de nouveaux modes de gouvernance, fondés sur un contrat social entre les acteurs publics et privés du territoire (Bertrand, op. cit). Il s'agit d'instituer un mode de pouvoir organisé autour d'un projet, en réunissant les diverses composantes de la société civile qui coopèrent avec les élus pour appliquer des orientations politiques élaborées et ratifiées ensemble.

61 La forme de gouvernance qui en résulte, se compose d'un ensemble d'organes ayant chacun, une place et un rôle spécifique et bien articulé entre eux. Au sein des communautés villageoises béninoises, notamment dans les départements des Collines et de l'Atlantique, différents systèmes de gouvernance sont rencontrés ces dernières années. Ceux-ci impliquent les populations locales, leurs responsables politico- administratifs, les partenaires au développement (ONG, services déconcentrés de l'Etat, autres bailleurs..). L'analyse de ces systèmes de gouvernance fait ressortir une participation des populations dans la conduite d'opérations de développement à travers divers dispositifs institutionnels. Il s'agit entre autres :

- des comités villageois de suivi ou de gestion ;

- le dispositif institutionnel des projets de territoire dans le département des collines ;

- les dispositifs des Comités Villageois de Développement (CVD) ou des Comités Villageois de Développement Local (CVDL), actifs respectivement dans les villages bénéficiant du Projet d'Activités Génératrices de Revenu (PAGER), et dans ceux faisant l'objet de la mise en œuvre du Projet de Gestion des Terroirs et des Ressources Naturelles (PGTRN) dans le département de l'Atlantique.

4.2. Mobilisation villageoise dans le cadre des comités de suivi et de gestion

62 L'émergence des comités de suivi et des comités de gestion a fait suite au constat généralisé du manque de participation des populations villageoises, dans les actions de développement initiées et conduites pour eux par les experts extérieurs à leurs communautés, sans leur implication réelle dans les démarches d'identification, de montage, de gestion et de suivi/évaluation desdites actions. Des évaluations faites vers la fin des années 1980 sur nombre de programmes initiés sur ces actions, ont donné des 
résultats globalement décevants. Ceci qui a fait prendre conscience aux décideurs et aux bailleurs de fond, à envisager une implication plus accrue des communautés villageoises dans les programmes liés à leur développement, à travers leur mobilisation depuis l'identification des actions jusqu'à leur évaluation, en passant le cas échéant par leur gestion.

63 Au départ présente dans les approches des ONG, l'organisation des bénéficiaires d'actions en comité de suivi, s'est généralisée par la suite dans les pratiques en cours dans les projets et programmes gérés sous la tutelles des ministères ou agences gouvernementales d'exécution.

Elle se retrouve dans le dispositif de mise en œuvre de plusieurs types d'actions dans les villages, dont les infrastructures (les salles de classe dans les écoles, les équipements d'adduction d'eau potable, les centres de santé, les pistes de dessertes rurales, etc.).

Au sein du comité de suivi d'action dans un village, se retrouvent souvent le chef de village ou son représentant, les responsables d'organisations paysannes (groupement villageois ou groupements à vocation coopérative), les représentants d'association de développement, les représentants de différents quartiers ou hameaux du village, les leaders d'opinions qui sont des sages ou des personnalités influentes du village, etc. Ce comité au sein duquel se trouvent représentées les différentes catégories socioprofessionnelles du village, sert d'interface entre la communauté villageoise et les intervenants extérieurs que sont les ONG, les programmes nationaux d'appui et d'autres partenaires. Au niveau du village, il organise la population et mobilise sa participation matérielle et financière à la mise en œuvre d'action.

comité de suivi dispose d'un bureau de direction en vue de sa coordination interne. L'ensemble des membres du comité sont responsables devant l'assemblée générale des villageois, à qui ils doivent rendre compte (souvent en réunion) sur les démarches menées dans le cadre de la mise en œuvre de l'action, depuis la phase d'identification. A la fin de la réalisation de l'action, le comité de suivi selon le cas cède la place à un comité de gestion, avec la possibilité de reconduction de certains membres compte tenu des compétences dont ils ont fait preuve. Ce cas concerne notamment les actions de réalisation d'équipements (systèmes d'adduction d'eau, équipement marchand tels les magasins, les hangars de marché, etc.). Il existe des villages où des comités de suivi sont reconduits d' une années à l'autre et d'une action à la suivante. La composition et le fonctionnement du comité de suivi mettent en relief, l'activation des relations entre divers types d'acteurs locaux. Le chef de village constituent le représentant du pouvoir local, au nom duquel il est parfois amené à prendre des engagements, et auprès duquel il doit rendre compte. Les représentants d'organisations locales (Groupement villageois, Groupement à Vocation Coopérative, association de développement ,..) traduisent dans leur implication, des degrés d'engagement de leurs structures mandataires. Les représentants des différentes agglomérations quant à eux, manifestent les engagements et implications de leurs populations. Tous ces éléments constituent des interlocuteurs du village en face des intervenants (partenaires et bailleurs), dans le processus de la mise en œuvre d'action. L'ensemble des acteurs villageois et des intervenant extérieurs constituent autour de cette dernière, un système local (villageois) d'action publique sous la coordination du comité de suivi.

67 L'approche des comités de suivi a été appropriée ces dernières années, par plusieurs programmes nationaux d'appui au développement au Bénin. C'est notamment, le cas des 
actions de construction d'infrastructures villageoises dans le cadre de l'Agence de Financement des Initiatives de Base (AGeFIB). C'est aussi le cas des actions du Programme d'Adduction d'Eau en milieu Rural (PADEAR).

68 Certains comités de suivi ont leur champ d'intervention plus vaste, dépassant le cadre villageois. C'est le cas par exemple des comités de suivi inter village, dans la conduite des actions de désenclavement (aménagement des pistes de desserte rurale). Les programmes de désenclavement conduits respectivement dans les Collines par l'ONG Suisse Helvétas et le Programme de Consolidation des Coopératives Agricoles (ProCoCa), constituent des cas illustrant cet aspect.

- Cas des actions pistes du programme Helvétas

Pour assurer la participation des populations de tous les villages riverains dans l'aménagement de chaque piste concernée par ce programme, celles-ci sont appelées à se concerter et à déléguer des personnes représentatives de chacun des villages, pour constituer un comité inter villageois de suivi. Les membres de ce comité sont chargés de mobiliser la participation physique et financière des populations dans chaque village, de suivre l'évolution des travaux et d'en rendre compte à chacune de celles-ci. Dans le cadre de l'entretien des pistes aménagées, ce comité cède à la fin des travaux la place à un Comité Inter villageois de Gestion des Pistes (CIGEP) dont les membres sont désignés selon la même procédure; quelques membres pouvant d'ailleurs être reconduits dans certains villages. Les démarches de montage et de mise en œuvre des actions mettent concertation et en coopération, les populations villageoises à (travers leur comité inter villageois de suivi), les autorités politico- administratives locales (chefs d'arrondissement, sous-préfet), les tacherons et professionnels des travaux publics et les animateurs du programme. Loin de constituer de simples représentants du pouvoir au plan local, les autorités politico- administratives constituent parfois de véritables forces de proposition au sein de ce dispositif d'acteurs institutionnels.

71 - Cas du volet piste du ProCoCa

72 Celui-ci est mis en œuvre dans le cadre d'une maîtrise d'ouvrage de l'Union Départementale des Producteurs (UDP) du Zou, avec l'appui de l'Agence Française de Développement (AFD). Dans le cadre de ces actions, le comité de suivi est constitué par les représentants de Groupement Villageois (GV) des villages riverains. Son fonctionnement est analogue à celui mis en place dans le cadre du programme Helvétas. Ce comité mobilise par le biais des GV, la participation financière des villages au cofinancement des actions. Les procédures de choix et de montage des actions, voient la participation des responsables des Unions Sous Préfectorales des Producteurs (USPP) concernées, aux côtés de ceux de l'UDP et des professionnels du domaine.

a. Points forts du dispositif comité de suivi

73 L'approche participative mobilisant les comités villageois de suivi, apparaît intéressante en matière d'implication effective des bénéficiaires d'actions de développement. Elle permet en effet aux villageois d'occuper un rôle plus central dans le choix de leurs priorités, la planification des actions qui en découlent et dans leur mise en œuvre. Elle favorise par ce biais, une appropriation de la démarche d'initiation et de mise en œuvre des actions de développement par ces acteurs à la base. Elle constitue de par ces éléments, un facteur de révélation des préférences en développement par les populations villageoises. 
74 Sur un autre plan, le dispositif du comité de suivi offre à la population villageoise un cadre organisationnel de regroupement, pour la conjugaison de ses énergies en vue de la recherche de solutions à des difficultés communes. Il constitue de ce fait, un élément de proximité organisationnelle pour les habitants d'un même village. Les comités de suivi inter villageois, en impliquant les représentants de différents villages dans un même cadre de concertation, contribuent à la proximité organisationnelle entre différentes populations villageoises.

75 En favorisant des concertions entre les autorités politico- administratives locales et d'autres représentants villageois, elle favorise dans une certaine mesure, le rapprochement des populations de leurs élus. Ainsi, tout en étant un outil de promotion de la gouvernance dans le village, elle favorise une décentralisation du processus de prise de décision au niveau villageois. Fort de ses succès, elle suscite l'intérêt de nombre de partenaires au développement. Sur proposition des ONG avec lesquelles ils sont en partenariat pour la mise en œuvre des actions, nombre de programmes gouvernementaux d'appui au développement systématisent la conduite de diagnostic villageois et la mise en place de comité villageois de suivi, avant le démarrage effectif d'actions de développement dans les villages béninois. C'est le cas notamment des programmes AGeFIB, PADEAR, etc.

b. Points faibles du dispositif

Le dispositif du comité de suivi, malgré les points fort soulignés, ne manque pas de faiblesse.

En effet le comité de suivi, même si dans le cas de certains types d'action, est remplacé par un comité pérenne de gestion, présente un caractère ad'hoc et son intervention reste en général, limitée à l'action pour laquelle elle est installée. Il ne s'inscrit pas toujours dans une démarche globale et durable de développement. Par ailleurs, le recours de plus en plus systématique à ce dispositif dans la procédure de conduite d'actions en milieu rural fait référence à un effet de mode, plutôt qu'à un besoin réel. Il n'est pas rare que le comité de suivi soit installé par le partenaire en développement $(\mathrm{ONG})$, pour répondre à des obligations méthodologiques du bailleur et que l'essentiel des initiatives et des décisions provienne de ce partenaire. Le comité de suivi se réduit dans ces conditions, à un outil d'aide à la sensibilisation et à la mobilisation (de la population) au service de l'opérateur en développement. A l'instar de cette approche, il existe dans certaines localités dans les départements des Collines et de l'Atlantique, des démarches plus globales dont les spécificités recommandent de les passer en revue.

4.3. Dispositif des projets de territoires dans le département des Collines.

Le dispositif des projets de territoires, qui s'inscrit dans la mise en œuvre d'un programme de développement local dans chacun des six communes du département des Collines, part d'un diagnostic villageois. Ce diagnostic était conduit pour la première fois en 1996, dans chaque village à l'intérieur de chaque commune. A cette occasion, toute la population villageoise est convoquée en assemblée générale, pour y effectuer le diagnostic global de développement du village. Au cours de l'assemblée, toutes les priorités de développement du village sont énumérées et hiérarchisées par elle. Pour le suivi des travaux de l'assemblée, la population villageoise choisit alors par désignation démocratique un représentant villageois. Celui-ci sera le porteur des priorités de son village, aux niveaux de décision et de concertation inter villageoises. 
Les représentants des différents villages d'une même commune se réunissent par la suite, pour mettre en cohérence et hiérarchiser l'ensemble des priorités de développement, définies par leurs populations respectives. Les priorités en développement dégagées à l'issue de cette assemblée des représentants, vont servir de base à l'élaboration du programme d'actions de la commune. Cette assemblée des représentants procède à la désignation démocratique d'un représentant, parmi ceux issus des villages de chaque arrondissement. L'ensemble de ces représentants d'arrondissement forme un organe de coordination et de concertation, appelé comité de territoire. Ce dernier est dirigé par un président et un secrétaire, choisis en son sein par ses membres.

Le comité de territoire organise des séances de concertation et de discussion avec les acteurs et partenaires du territoires (organisations locales, responsables d'ONG, responsables de projets et de services déconcentrés de l'Etat, ..), afin de traduire les priorités de l'ensemble des populations du territoire communal, en actions concrètes de développement. Il se charge de la planification des actions en programmes d'actions (annuel ou pluriannuel). Les présidents des comités de territoires de l'ensemble des six communes du département des Collines, forment avec les six maires de ces dernières un organe de décision au niveau départemental, appelé Conseil de Développement Territorial (CDT). Le CDT se réunit périodiquement (au moins une fois l'an), pour étudier et valider le programme d'actions de chaque territoire (commune) en séance plénière de présentation. Au cours de cette séance, le président et le secrétaire de chaque commune présentent aux autres membres du CDT, le contenu du programme d'actions de leur territoire. A l'issue de la présentation suivie de débat, le CDT dont les sessions sont dirigées par un président et un secrétaire démocratiquement élus, prend une décision relative au financement de chaque action présentée par chaque territoire. Des observations sont éventuellement faites, pour favoriser une mise en œuvre adéquate des actions sur les territoires concernés.

81 Les actions réalisées sur le territoire communal, relèvent de la maitrise d'ouvrage communal. Dans le plan de financement, $20 \%$ du coût total de chaque action est prise en charge par le budget communal alors que les $80 \%$ restant sont jusque là supportés par les subventions des bailleurs (exemple: Agence Française de Développement). La coordination et le suivi global de la mise en œuvre du programme d'actions dans chaque commune, est assurée par le comité de territoire. Toutefois, dans le cadre du montage et de la mise en œuvre d'une action dans un village de la commune, la population de ce dernier choisit en son sein des personnes représentatives, pour constituer un comité villageois ad' hoc de suivi. Au besoin, après la réalisation de l'action, elle choisit d'autres personnes pour sa gestion.

Le comité de territoire organise au moins une fois l'an, une Assemblée Générale du Projet de Territoire (AGPT) au cours de laquelle, il est tenu d'informé les représentants de tous les villages de la commune, sur la mise en œuvre du programme d'actions. Les représentants villageois sont à leur tour, chargés de restituer les informations de l'AGPT à leurs populations respectives, réunies en assemblées. Dans le même souci d'informer la population communale, le comité de territoire organise et met en œuvre, en partenariat avec les radios locales un plan de communication sur le projet de territoire et la mise en œuvre de son programme d'actions. Ceci converge avec les propos de GUIGOU sur le fait que, le développement local produit et recherche de l'information, et organise sa propre communication.

a. Points forts du dispositif 
83 Le dispositif projet de territoire, en favorisant des concertations directes des communautés villageoises dans le diagnostic de leurs milieux et dans la recherche de solutions, constitue un outil de révélation des préférences en développement par les populations concernées. Le processus de désignations directe et indirecte de représentants qui l'accompagne du niveau villageois au niveau départemental, en passant par l'arrondissement et la commune, en fait un instrument de promotion de la démocratie participative. Il donne lieu à un regroupement des représentants de tous les villages d'une même commune, dans une organisation locale de validation (assemblée générale de projet de territoire). Il met en place de ce fait une organisation du genre réseau, qui relie les différents villages de la commune pour une même cause, le projet de territoire. Il favorise de ce fait une proximité organisationnelle entre les acteurs villageois de la commune.

Pour ces derniers et d'autres types d'acteurs (organisations locales, structures d'appui, entrepreneurs,..), qui sont impliqués dans la démarche de projet de territoire, ce dernier est devenue une référence dans le département des Collines, ce qui les met dans une situation de proximité institutionnelle. Dans le Conseil de Développement Territorial (CDT), les élus locaux et les représentants de chaque commune du département, sont réunis dans un même cadre de concertation, permettant ainsi une implication des populations dans les prises de décisions sur leur développement. Il s'agit là d'un élément en faveur de la décentralisation. Il y a ici le lieu de souligner le mérite du dispositif du projet de territoire dans le département des Collines, de s'articuler de par les structures mises en place et de par leur fonctionnement, de s'insérer dans le dispositif de la décentralisation au Bénin.

Cette démarche donne lieu à un dispositif de fédération des initiatives en développement de l'ensemble des villages d'une même commune. Elle permet la conjonction des énergies de plusieurs acteurs du système local d'action publique, relatif au territoire communal. Elle constitue un outil de gouvernance locale, aux niveaux communal et même départemental dans les Collines.

b. Points faibles du dispositif

$\mathrm{Au}$ stade actuel, le dispositif du projet de territoire est loin de parvenir à une coordination de toutes les initiatives en développement à l'échelon communal. En effet, les localités des Collines, à l'image de l'ensemble de la zone rurale béninoise, constituent des terrains d'intervention de plusieurs opérateurs en développement, dont certains s'intéressent à la fois aux mêmes populations et aux mêmes difficultés, avec des approches parfois semblables, sans qu'il n'existe une concertation, voire une réelle coordination entre elles. Des initiatives des nouveaux élus communaux qui viennent d'être installés entre février et mars 2004 au Bénin permettront peut être, d'affirmer la responsabilité des collectivités locales dans le sens de cette coordination.

87 Par ailleurs, dans le montage financier des actions du projet de territoire, la participation des bénéficiaires villageois n'est pas directe. Il est supposé que, la participation du budget communal à raison de $20 \%$ du coût des investissements constitue une manière d'impliquer les populations dans le financement de leur développement. Une telle façon de procéder n'est pas intégrée dans les pratiques en cours chez les populations béninoises en matière de financement de leur développement. En effet, dans le cadre de celles-ci, les populations villageoises procèdent souvent, par cotisation directe des ménages, contribution financière de leurs propres organisations économiques (GV, GVC, ..), ou par souscription volontaire de personnes membres de leurs associations villageoises de 
développement. Elles ont de ce point de vue, du mal à faire la différence entre la pratique du projet de territoire et celle traditionnel du « développement purement assisté » par l'Etat ou les bailleurs. Ceci présente des risques réels par rapport à l'appropriation entière des actions réalisées, car les populations villageoises ont tendance à assimiler ces réalisations, comme étant celles de l'opérateur qui anime la conduite du projet de territoire, ou celles de bailleurs impliqués.

4.4. Dispositifs des CVD et des CVDL dans le département de l'Atlantique.

Dans le département de l'Atlantique, le dispositif des Comités Villageois de Développement (CVD) se retrouve actuellement dans les villages Lissègazoun (Allada) et Sè (Toffo), dont les habitants bénéficient des appuis du Projet d'Activités Génératrices de Revenu (PAGER) . Il se retrouve sous la forme de Comité Villageois de Développement Local dans le village Adjadji-Kossoé (Allada), dont la population est appuyée par le Projet de Gestion du Terroir et des Ressources Naturelles. - Le Comité Villageois de Développement (CVD)

L'initiative des CVD dans les villages Lissègazoun et Sè est venue de la volonté du Projet d'Activité Génératrice de Revenu (PAGER) et de ses ONG prestataires de services, de dynamiser les villages dans lesquels ils interviennent, en favorisant l'émergence de structures communautaires au sein des populations villageoises, afin qu'elles portent et approprient les actions d'aménagement d'infrastructures socio-économiques, qu'ils les aident à mettre en œuvre. L'idée au départ était aussi de former par ce biais, ces populations villageoises à une approche de la gouvernance locale qui pourrait leur être utile, après le démarrage effectif du processus de la décentralisation au Bénin.

Plusieurs responsables du PAGER appréhendent les CVD comme des structures du genre " association de développement". Il s'agit en fait d'un noyau de leaders et de représentants de la population villageoise, autour duquel se mobilise celle-ci en vue du développement de son milieu. Vu dans cet esprit, le CVD avec l'ensemble de sa population peut être perçu comme une association villageoise de développement. Les membres du CVD dont l'effectif varie (9 à 11) selon la taille du village, sont désignés par un collège électoral composé de représentants de différents quartiers ou agglomérations du village. Au départ, durant quelques jours précédant la désignation des membres du CVD, des séances de sensibilisation sont menées par les animateurs du PAGER dans toutes les agglomérations du village, sur la composition et les fonctions du CVD ainsi que, sur ses enjeux pour le développement du village. Il est alors demandé à chaque quartier du village, de choisir de façon démocratique ses représentants pour participer dans le cadre d'un collège électoral, à la désignation des membres du CVD. Ces représentants dont le nombre est le même pour chaque quartier, sont souvent désignés par consensus. A la réunion de désignation des membres du CVD par le collège électoral, l'organisation veille à ce que, tous les quartiers soient représentés dans le comité. Le CVD comporte un certain nombre de poste de direction. Il est admis dans les principes que, le chef du village en fasse partie sans en être le président. Le CVD constitue une sorte d'interface entre la population villageoise et ses partenaires en développement, notamment les structures d'intervention extérieures (ONG, projets, administration, bailleurs,..).

Ce comité organise chaque année une assemblée villageoise de diagnostic, pour définir et actualiser les priorités en développement du village. A cette assemblée, la population procède à une planification des actions à mener dans l'année à venir. Cette planification sert de base à l'élaboration d'un programme d'actions annuel par le CVD. A cet effet, il organise des concertations avec les organisations locales (organisations paysannes, 
associations, ..), les entrepreneurs et les partenaires au développement du village, pour le montage et la mise en œuvre des programmées. Le CVD mobilise la participation physique et financière des villageois à la mise en œuvre des actions. Elle constitue un comité permanent de suivi des actions de développement, à réaliser dans le cadre du PAGER dans le village. Pour le cas de la réalisation des actions à caractère marchand (magasins, ateliers, marchés, point d'eau, ..), le CVD met en place dans chaque cas un comité spécifique de gestion. Il assure la coordination de l'ensemble des comités de gestion mis en place dans le village. Le CVD réunit périodiquement dans l'année, la population villageoise en assemblée et lui rend compte de la mise en œuvre du programme d'action.

a. Points forts du dispositif du CVD

Le dispositif CVD apparaît comme un outil de révélation des préférences en développement des populations villageoises. En effet, sous la coordination de ces comités, les populations villageoises procèdent au diagnostic de leur situation et proposent des pistes d'actions pour leur propre développement. Le cadre du CVD permet à la population villageoise, de participer même directement à la planification des actions qu'elle se propose.

Dans ce dispositif, la population villageoise et ses représentants se concertent dans un même cadre que son chef de village. Il constitue un facteur de proximité organisationnelle entre les villageois. Il favorise aussi ainsi le rapprochement des populations avec leurs chefs de village qui sont en fait, des élus de ce niveau. Il permet aussi à la population villageoise, de désigner de façon indirecte des représentants, pour la coordination de ses opérations de développement. Il constitue donc dans une certaine mesure, un facteur de promotion de la démocratie et de la décentralisation de la prise de décision à l'échelon villageois. Par son intervention, la population villageoise est mobilisée pour sa participation physique et financière directe à son développement. La possibilité de planification des opérations directement par la population au niveau villageois et la participation directe de celle-ci à leur financement, renforcent le degré de proximité et de participation des villageois dans la conduite de leur développement. Cet élément constitue un atout qui valorise le dispositif CVD par rapport au dispositif du projet de territoire, du point de vue maîtrise d'ouvrage et appropriation du développement par les bénéficiaires directes.

b. Point faible du dispositif

Une limite par rapport au dispositif du projet de territoire est l'étroitesse du niveau de coordination villageoise face au niveau communal. Une conduite du processus du développement local dans plusieurs villages d'une commune, ou même d'un arrondissement, sans la mise en place d'un mécanisme de coordination inter villageois, risque de conduire à une situation d'incohérence et de sous- optimalité dans la gestion des moyens mobilisés.

Le dispositif du Comité Villageois de Développement Local prend en compte un tel mécanisme de coordination.

- Le Comité Villageois de Développement Local (CVDL)

L'initiative du CVDL vient du Projet de Gestion des Terroirs et des Ressources Naturelles (PGTRN), de doter les villages où il intervient, de structures communautaires dynamiques pour porter les actions, qu'il aide les populations à mettre en œuvre. Dans ce cas-ci, les actions sont surtout orientées vers la gestion des ressources naturelles et de 
l'environnement du terroir villageois. Les membres du CVDL sont choisis lors d'une assemblée générale par toute la population villageoise. La même assemblée se réunit pour faire le diagnostic du village et proposer des pistes de solutions, pour son développement.

Les membres des CVDL de tous les villages d'un même arrondissement se rencontrent, pour constituer un Comité d'Arrondissement de Développement Local (CADL). Dans ce cadre, chaque CVDL désigne un de ses membres, pour le représenter au niveau de l'arrondissement. Les CVDL et le CADL ont chacun, un organe de direction dont les éléments sont désignés démocratiquement, par leurs membres respectifs. L'ensemble des CVDL du même arrondissement et le CADL se rencontrent au départ pour réaliser la planification pluriannuelle, sur la base des pistes d'actions proposées par les populations lors des diagnostics villageois. Ils se retrouvent également au début de chaque année pour la planification annuelle des actions.

100 Les travaux de planification voient la participation de tous les chefs de village et du chef d'arrondissement. Avant la séance de planification, chaque CVDL se concerte avec le chef de son village pour harmoniser les points de vue. Ils se rencontrent également après cette séance, pour échanger sur la suite à donner aux résultats issus de la planification.

101 A l'issue de celle-ci, chaque CVDL organise en assemblée villageoise, une séance de restitution des résultats à sa population. De même, le CADL organise périodiquement des séances de communication à la radio locale, sur l'évolution de la mise en œuvre des actions planifiées. Outre les fonctions énumérées, le CVDL coordonne dans son village, l'élaboration du budget d'activités annuel, la conduite d'études de faisabilité sur les actions planifiées, l'auto- évaluation d'activités tous les semestres, l'évaluation annuelle... Le CADL se charge quant à elle, de la coordination des activités des CVDL sur l'ensemble de l'arrondissement. Les actions mises en œuvre depuis l'année 2001 par le PGTRN avec le partenariat des CVDL et du CADL, ont concerné pour le cas par exemple du village d'Adjadji-Kossoé, la construction d'un centre de formation, la clôture de la forêt sacrée, la promotion (formation et appui à l'installation) de huit groupements de pépiniéristes, la promotion de trois groupements d'apiculteurs dans le village.

a. Points forts du dispositif du CVDL

102 Le dispositif du CVDL présente des caractéristiques et des atouts analogues à celui du CVD. Les principales différentes résident au fait que, les représentants sont ici choisis au niveau villageois en assemblée villageoise directement par la population, alors que ce choix se fait par un collège électoral dans le cas des CVD.

103 Il existe ici un niveau de coordination à l'échelle de l'arrondissement. En effet, a planification des actions se fait par les représentants au niveau de l'arrondissement ; mais tout comme dans le cas des CVD, le diagnostic se fait par tous les villages. Il apparaît ainsi que, ce dispositif favorise aussi la révélation des préférences en développement par les populations villageoises concernées. Il constitue un facteur de proximité organisationnelle non seulement entre les habitants d'un même village, mais aussi entre les représentants et élus de différents villages du même arrondissement. Il représente à l'instar des dispositifs projets de territoire et CVD, un instrument de promotion de la démocratie et de la gouvernance participative. Il constitue aussi un élément en faveur de la décentralisation dans les localités où il est mis en œuvre.

b. Point faible du dispositif

104 Le dispositif du CVDL s'applique à un niveau d'arrondissement, ce qui limite son efficacité dans le cadre d'une coordination du développement à l'échelle communale. A cet égard, il 
présente à l'instar du dispositif du CVD, un encrage limité par rapport au processus de la décentralisation au Bénin. En effet, le seul niveau actuel de la décentralisation au Bénin est communal, alors que les schémas de développement proposés respectivement par les dispositifs CVD et CVDL sont respectivement adaptés aux niveaux du village et de l'arrondissement.

Conclusion

Il apparaît au vu des expériences qui précèdent, que différentes formes de gouvernance s'inscrivent dans les systèmes locaux d'action publique en présence dans les localités rurales béninoises, notamment celles des départements de l'Atlantique et des Collines. Ces formes de gouvernance font ressortir un dynamisme notable des communautés villageoises, en terme

de révélation de leurs préférences en développement, et de la satisfaction des utilités liées à celles-ci. Dans ce cadre, les populations villageoises se mobilisent selon la localité, suivant des dispositifs de comité de suivi, comité villageois de développement (cas de l'Atlantique), de projet de territoire (cas des Collines). Il s'agit des cadres de concertation au sein desquels, les principes de discussion à palabre et de consensus amènent les populations à s'entendre autour des priorités communes de développement. Ainsi, loin d'être des choix purement individuels, ni leur somme arithmétique, les préférences en développement révélées par les populations locales dans le cadre de ces dispositifs, sont des préférences collectives. Dans l'expression de ces priorités, le poids des leaders (sages, chefs villages, responsables d'organisations paysannes, représentants, ..) apparaît primordial. En effet, en milieu africain et notamment en zones rurales béninoises, les leaders sont les personnes en qui les populations locales se confient en matière de décisions collectives.

La mobilisation des populations locales à l'intérieur de ces cadres de concertation favorise entre elles, l'expression d'une proximité organisationnelle. Elle permet également le rapprochement entre ces populations et leurs leaders, en particulier les chefs de village et même les élus locaux. Etant des outils de rapprochement des populations des niveaux de prise de décisions les concernant, ces dispositifs favorisent leur bonne implication dans celles-ci. Ils constituent donc des outils de gouvernance favorables à l'enracinement du processus de la décentralisation.

108 Des outils nécessaires à l'apprentissage de la bonne gouvernance locale existent donc dans les localités rurales béninoises; mais faudrait-il que les nouveaux élus puissent les valoriser dans le cadre de leurs missions. Dans le département des Collines par exemple, une méthodologie d'élaboration du plan de développement communal peut s'inspirer de la démarche des projets de territoires. Le dispositif institutionnel de ces derniers peut permettre aux élus, de prendre en compte les préférences des populations des différents villages, en restant proches d'elles dans la démarche et en respectant le cadre tracé par la loi.

109 Dans le département de l'atlantique, le dispositif du CVD peut aider le chef de village à faire émerger les préférences en développement de sa population, et à les faire prendre en compte par le conseil communal, par le biais de son chef d'arrondissement. Ce rôle peut aussi être joué par le CVDL qui s'apparente au CVD au niveau villageois. Le CADL, organe de coordination des CVDL peut constituer un partenaire privilégié du chef d'arrondissement. La méthodologie en cause dans la démarche CVD en général, peut inspirer les élus communaux du département de l'atlantique, dans la conception et la mise en œuvre de plans adéquats de développement des communes. D’autres expériences 
non moins enrichissantes existent dans d'autres départements du Bénin. Il revient alors aux élus de prendre connaissance des outils utilisés par celles-ci, et d'y puiser des éléments pour une gestion et une coordination harmonieuse des actions de développement sur leurs territoires respectifs.

L'attribution de nouvelles responsabilités aux collectivités locales et la responsabilisations des populations à la base dans la conduite du processus de développement sur leurs territoires, appellent à de nouveaux modes de collaboration et coordination des acteurs et des interventions au plan local. La recherche des formes de gouvernance locale en fonction des spécificités de chaque milieu, apparaît dès lors comme une nécessité.

La richesse des expériences en la matière sur certains territoires ruraux au Bénin, constitue une potentialité sur laquelle les nouveaux élus locaux de ce pays peuvent activer, pour combler une des principales attentes des populations à leur endroit. Le dispositif des projets de territoire dans le département des Collines et celui des CVD dans l'Atlantique, représentent à cet égard, des éléments de gouvernance qui peuvent être exploités et adaptés à la situation d'autres milieux.

\section{BIBLIOGRAPHIE}

BELLET, COLLETIS, LUGBY, PECQUEUR, RALLET, TORRE, (1992), « Et pourtant ça marche ! Quelques réflexions sur l'analyse du concept de proximité », in chronique : Etudes empiriques, Revue $d^{\prime}$ Economie industrielle, $\mathrm{n}^{\circ} 61,3^{\text {ème }}$ trimestre.

BERTACCHINI Y. (2002) : «Concertation territoriale et politique territoriale concertée », Laboratoire Le Pont, Département Services et Réseaux de Communication, Université de Toulon et du Var, $6 \mathrm{p}$.

BERTRAND N., GORGEU Y., MOQUAY P. (2001) : «Intégration des formes de proximité dans la gouvernance locale », Les troisièmes journées de la proximité « Nouvelles croissance et territoires », Paris, 13 et 14 Décembre 2001, 18p.

BESLAY C. et al. (1998) : La construction des politiques locales. Reconversions industrielles et systèmes locaux d'action publique. Paris, France, L'Harmattan, 220 p.

BIRD R. et VAILLANCOURT F. (1997) : Décentralisation financière et pays en développement : concepts, mesure et évaluation, Cahier de recherche, Université de Montréal.

BODY-GENDROT S. et Le GALES P. (1997) : « Qu'est-ceque la gouvernance locale ? », in CARRE J. et BODY-GENDROT S., Gouvernance locale, pauvreté et exclusion dans les villes anglo-saxones. Centre d'Etudes des Relations Interculturelles. Frontière.

BONVIN (1994) : Promouvoir la maîtrise locale et régionale du développement. Une démarche participative à Madagascar, Documents de travail de l'OCDE, $\mathrm{N}^{\circ} 36$, Paris.

BROUSSEAU E. (1993) : L'économie des contrats ; technologie de l'information et coordination interentreprise. Ed Presse Universitaire de France.,368 p. 
COLLETIS G., GILLY J.P. (1999), « Construction territoriale et dynamiques économiques », Sciences de la Société, octobre.

COMMONS J. R. (1934) : Institutionnal economics, Madisson, Wis, University of Wisconsin-Press.

DREGER M. (2000) : Le dispositif du projet de territoire du programme de coopération décentralisée Picardie-Collines et sa compatibilité juridique avec la réforme de l'administration territoriale de 1999 du Bénin. Rapport de stage. Avril .67p.

DERYCKE P-H et GILBERT G. (1988) : Economie publique locale, Paris, Economica. 308p.

FOIRRY J.-P. (1997) : Economie publique: Analyse économique des décisions publiques. Paris.

Hachettes. $160 \mathrm{p}$.

GILLY J.-P. et TORRE A. (2000) : Dynamiques de proximité. Paris. L'Harmattan.

GILLY J.P et WALLET F. (1998) : «Proximité, hybridation et gouvernance locale : contribution à l'analyse des dynamiques territoriales », colloque ASRDL, Puela, Septembre 1998.

GUIGOU J.L. (1986) : «Le développement local : espoirs et freins », in GUESNIER B. (1986) : Développement local et décentralisation. Editions Régionales Européennes.

HOUNMENOU B. (2001) : Dynamiques de développement économique et systèmes productifs locaux en territoires décentralisés des pays en développement. Mémoire de DEA en développement soutenable intégré (Développement économique et Social), Université de Versailles Saint Quentin en Yvelines, France, 89 p.

HOUNMENOU B. (2002) : « Nouvelles politiques de développement local dans les pays en développementCas du milieu rural au Bénin. » Communication au colloque Syal (Systèmes AgroAlimentaires Localisés) ; Montpellier, du 16 au 18 octobre 2002. 18 p.

JARRET M.-F. et MAHIEU F.-R. (1998) : Economie publique :Théories économiques de l'interaction sociale. Paris. Ellipses. 123 p.

LAHAYE N. (1999) : «Gouvernance territoriale et espace d'intérêt public : l'enjeu d'un développement durable territorial », LEREPS, Université des Sciences SocialesManufacture des Tabacs- Ecole- chercheur Economie spatiale et régionale, 8, 9, 10 décembre 1999- Le Croisic. 24 p. Le GALES P. (1995) : « Du gouvernement des villes à la gouvernance urbaine ». Revue française des sciences politiques. 45 (1) : 58- 95.

Le GALES P. (1993) : Politique urbaine et développement local- Une comparaison franco-britannique. Paris, France, L'Harmattan.

LINDAHL E. (1919) : “Just Taxation. A positive Solution”, in MUSGRAVE and PEACOCK, eds, Classics in the Theory of Public Finance. London, MackMillan, 1958.

MALIBEAU A. (1994) : Le système local en France. Paris, Montchrestien, coll. Clefs.

MALINDI E. (1998) : « Les composantes socio-économiques du développement local et participatif en Afrique noire ", communication au colloque international sur les composantes locales, régionales, mondiales du développement des Nations. Antagonisme ou complémentarité?, Poitiers, 28-30 avril.

MEULDERS, THYS-CLEMENT et WILLIAMS (1982) : «Le fédéralisme fiscal et les fonctions des pouvoirs publics »- Note ronéotée. DULBEA, juin. $20 \mathrm{p}$.

MORIN R. et LEMIEUX A. (1999) : « Développement local et ancrage territorial des entreprises dans les quartiers d'une ville-centre », in Revue d'Economie Régionale et Urbaine, $\mathrm{N}^{\circ} 5$. 
D. OSBONE (1988) : Laboratories of democracy (a new breed of governor creates models for National Growth), Havard Business School Press, Massachusetts.

PADIOLEAU J. G. (1991) : « L'action publique moderniste après la décentralisation », Politique et management public, vol.9, $\mathrm{n}^{\circ} 4$.

PARKS R. B., OAKERSON R.J.(1989) : "Metropolitan organisation and governance. A local public economy approach”, Urban affairs quarterly, vol.25, n²1, pp.18-29.

PECQUEUR B. (2000) : Le développement local : pour une économie des territoires. Paris. Syros.

PERROUX F. (1961) : «Qu'estce que le développement local ? », Etudes, janvier.

REQUIER-DESJARDINS D. (2000) : « Décentralisation et dynamiques locales de production dans les pays en développement », C3ED/UVSQ.

SHAH A. (1998) : Balance, Accountability, and Responsiveness : Lessons about Decentralization, World Bank, Washington .

SCHNEIDER H. (1999) : « Gouvernance participative : Le chaînon manquant dans la lutte contre la pauvreté », Cahier de Politique Economique n¹7, OCDE.

STONE C. N. (1993) : Urban regimes and the capacity to govern- A political economy approach.

TEISSERENC P. (1994) : « Politiques de développement local. La mobilisation des acteurs », Sociétés contemporaines, $\mathrm{n}^{\circ} 18 / 19,1994$, p.187- 213.

TORRE A. (2000) : « Economie de la proximité et activités agricoles et agroalimentaires », In Revue d'Economie Régionale et Urbaine, 2000- n³. pp 408-425.

UNAL M. (2000) : «Les mots clés d'une circulaire : contribution sémantique et méthodologique à la maturation des intercommunalités au regard des perspectives données par le rapport Mauroy », Colloque Réseau de ville, Paris, 7 décembre.

WALLET F. (1997) : «Gouvernance locale et action publique locale : l'exemple des processus de reconversion industrielle », Communication au colloque de l'ASRDLF, Lille 1-3 septembre, 33 p.

WALLISER B. (1989) : «Théorie des jeux et genèse des institutions », Troisième colloque des associations Charles Guide pour l'étude de la pensée économique. Emergence et fondements des concepts d'équilibre en économie, Strasbourg, 21 - 22 septembre.

WICKSELL K. (1896) : “A New Principle of Just Taxation”, in MUSGRAVE and PEACOCK, eds, Classics in the Theory of Public Finance. London, MackMillan, 1958.

\section{ANNEXES}

\section{LISTE DES SIGLES}

AFD : Agence Française de Développement

AGeFIB : Agence de Financement des Initiatives à la Base

AGPT : Assemblée Générale de Projet de Territoire

CADL : Comité d'Arrondissement de Développement Local

CDT : Conseil de Développement Territorial

C3ED : Centre d'Economie et d'Ethique pour l'Environnement et le Développement 
CIGEP : Comité Inter villageois de Gestion des Pistes

CVD : Comité Villageois de Développement

CVDL : Comité Villageois de Développement Local

DEA : Diplôme d'Etudes Approfondies

GV : Groupement Villageois

GVC : Groupement à Vocation Coopérative

ONG : Organisation Non Gouvernementale

PADEAR : Programme d'Adduction d'Eau en milieu Rural

PAGER : Projet d'Activités Génératrices de Revenu

PGTRN : Projet de Gestion des Terroirs et des Ressources Naturelles

ProCoCa : Programme de Consolidation des Coopératives agricoles

SYAL : Systèmes Agroalimentaires Localisés

UDP : Union Départementale des Producteurs

USPP : Union Sous Préfectorale de Producteurs

\section{RÉSUMÉS}

Les politiques de développement en cours dans plusieurs pays du sud, notamment dans ceux de l'Afrique sub-saharienne ont amorcé un nouveau tournant à partir de la fin des années 1980. A la faveur de l'instauration du climat de démocratie dans plusieurs de ces pays à partir de cette période, nombre d'entre eux ont procédé à de profondes réformes, donnant à la société civile et aux populations à la base, un droit de regard plus important dans la conduite des affaires les concernant. L'une des manifestations les plus importantes de ces réformes se traduit par la mise en œuvre de la décentralisation. Ce processus vise entre autres, la prise en compte des réalités locales et la responsabilisation des communautés à la base dans la gestion de leur développement. Il s'agit en d'autres termes, de favoriser le renforcement des capacités des populations locales, en vue de leur permettre de prendre en charge de façon durable, la gestion de leurs propres affaires dans le cadre de la gouvernance participative. Amorcé en janvier 1993, avec les états généraux de l'administration territoriale, le processus de la décentralisation au Bénin n'a connu sa phase de concrétisation qu'avec les élections et l'installation des élus locaux en début 2003. En prélude à l'avènement de la décentralisation, plusieurs localités béninoises font l'objet, depuis le milieu des années 1990, d'expériences d'appui au développement des communautés locales. C'est le cas notamment, des villages des départements de l'Atlantique et des Collines. A travers ces expériences, se mettent en place, divers dispositifs de gouvernance locale au sein des systèmes locaux d'action publique relatifs aux localités rurales. Il s'agit notamment, des comités villageois de suivi ou de gestion, des dispositifs de comités villageois de développement dans le département de l'Atlantique, et du dispositif des projets de territoire dans les Collines. En leur permettant de révéler leurs préférences collectives de développement, la concertation et la mobilisation des populations locales au sein de ces dispositifs favorisent leur proximité des niveaux de prise de décisions les concernant.

The currency development policies in many southern countries, especially in those of subSaharan Africa have started a new turn, by the end of the years 1980 . With the favor of the 
democracy climate in many of those countries from that period, number of them have proceeded to the deep reforms, giving to the civil society and the basic populations, an important right of glance in the management of the matters which concern them. One of the important manifestations of those reforms, is expressed by the implementation of the decentralization process. Otherwise, it is also to favor the local populations capacities reinforcement, in order to make them able to well support their own matters management, in the frame of the participative governance, through the decentralization process. Begun in January 1993 with the general statement of territorial administration, the decentralization process in Benin, has known its operational step with the election and the installation of the new local elected, about the beginning of the year 2003. Early, since the middle of the years 1990, in many Benin localities, local communities development experiences have been taking place, in order to prepare populations to the decentralization advent. It's especially the case of some villages in Atlantic department and all of those in Collines. Through those experiences, various systems of local governance are taking place in rural areas. It's the case of "village committees of monitoring or management", "village committee of development" system in Atlantic region, and the "territory project" system in Collines region. By allowing them to reveal their common preferences of development, the consulting and the mobilization of the local populations inside those systems favor their proximity to the level at which the decisions concerning them are taken.

\section{AUTEUR}

\section{BERNARD G. HOUNMENOU}

Formation : Bernard G. Hounmenou est ingénieur agro-économiste, titulaire d'un DEA en développement soutenable intégré (option, développement économique et social) de l'université de Versailles SQY. Il poursuit son doctorat depuis octobre 2001 à ce jour, au Centre d'Economie et d'Ethique pour l'Environnement et le Développement (C3ED) de l'université de Versailles - Saint Quentin en Yvelines sur le thème : « Dynamiques locales de développement économique en territoires décentralisés des pays en développementcas des territoires ruraux du Bénin ». (C3ED/ Université de Versailles Saint Quentin en Yvelines / France), 47, Bd VAUBAN- 78047 GUYANCOURT CEDEX, hbenaf@yahoo.fr 